\title{
Challenges in Low Voltage Imaging in the FE-SEM
}

Raynald Gauvin

Department of Materials Engineering, McGill University, M. H. Wong Bldg, 3610 University Street, Montreal, H3A 2B2, Quebec Canada.

The Field Emission Scanning Electron Microscope is now reaching a new era. This is the era of imaging with electron energies below $1 \mathrm{keV}$, that can be as low as $50 \mathrm{eV}$, with spatial resolution of the order of the $\mathrm{nm}$. This paper intends to show the possibility of imaging nanomaterials in these energies with a description of the challenges that will have to be overcome in order to understand this new revolution in imaging. As an example, pictures of tin-palladium particles deposited on multiwall carbon nanotubes and obtained at $200 \mathrm{eV}$ are shown in figure [1] with 2 different imaging modes of electrons. The details on the fabrication of the nanomaterials are described by Probst et al. [1]. These pictures were taken on the Hitachi SU-8000 FE-SEM that has 3 secondary electron detectors where 2 are through the lens (TTL). These 2 TTL detectors allow different imaging modes through various modes of energy filtering. The first mode shows contrast that seems to be related to atomic number contrast while the second seems to be more related to topographic contrast. By playing with the settings of these detectors, complementary information can be obtained from the same material with spatial resolution of the order of the $\mathrm{nm}$ at electron energy of $200 \mathrm{eV}$.

However, important scientific challenges will have to be overcome in order to understand quantitatively electron images obtained below $1 \mathrm{keV}$ of incident electron energy. The modeling of electron scattering in solids is more difficult to perform because the continuous slowing down approximation of the stopping power starts to fails at these low energies, as well as the inelastic cross-sections. This modelling must include a complete description of the Bethe surface that relates the oscillator strength to energy loss and momentum transfer. This is already a difficult exercise for isolated atoms where exchange effects must be included. It is even more in the case of solids where solid state effect on the band structure of valence electrons must be included in the modelling. The lack of good experimental data on SE and BSE yield at low energy is also needed to validate the models. Knowledge of the detection efficiency of these new electron detectors as well as the electro-magnetic field in the microscope to understand the selection process of the SE and BSE are also required.

\section{References}

C. Probst, H. Demers, R. Gauvin (2008), Microscopy \& Microanalysis, Supp. 2, p. 1202. 

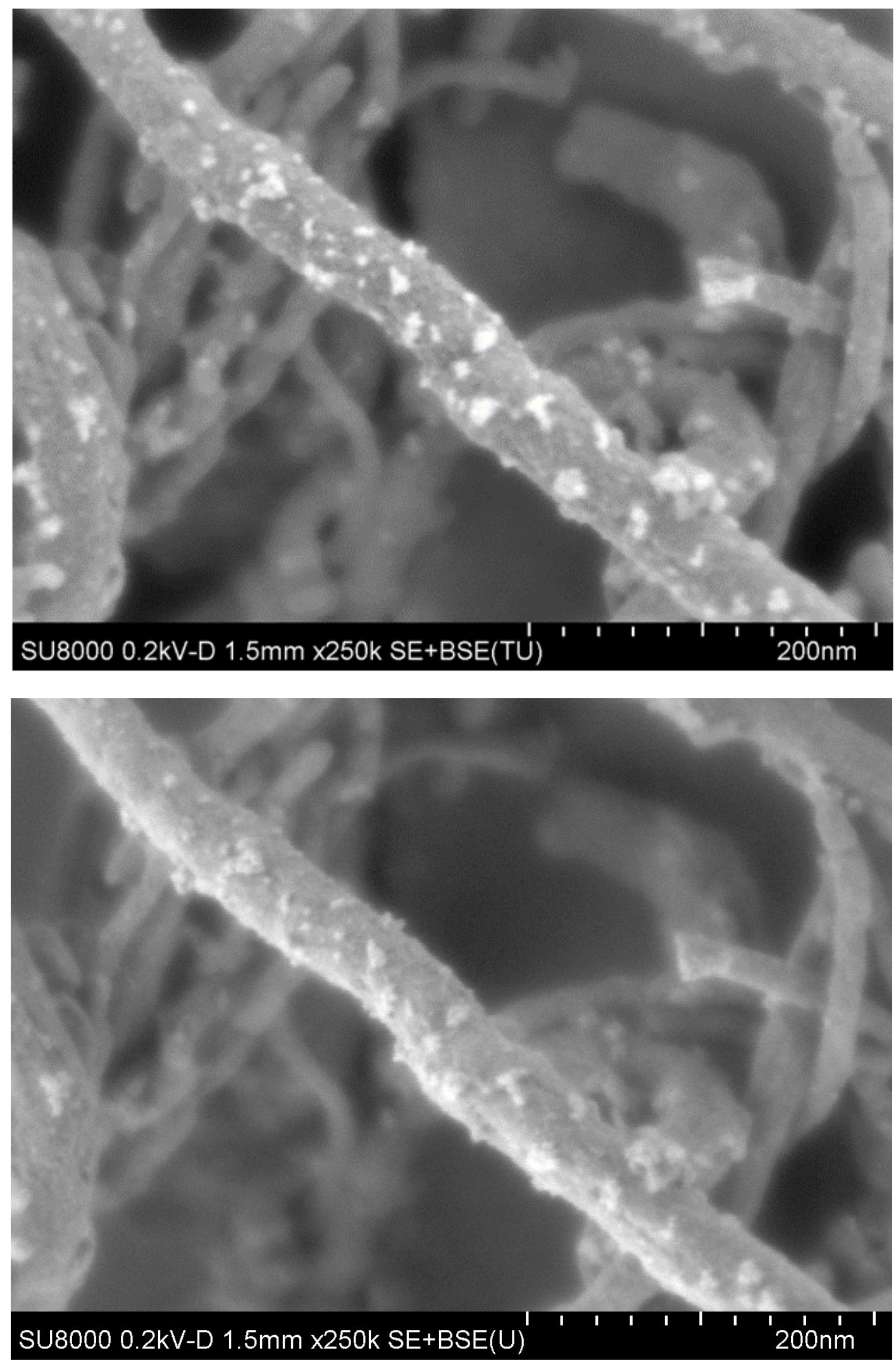

Figure [1] Top figure: TU Detector. Botton figure: U Detector. 